\title{
Short and slim nacelle design for ultra-high BPR engines
}

\author{
Matthew Robinson ${ }^{1}$, David G. MacManus ${ }^{2}$, Kelvin Richards ${ }^{3}$ \\ Cranfield University, Cranfield, United Kingdom, MK43 OAL \\ and \\ Christopher Sheaf ${ }^{4}$ \\ Rolls-Royce plc, P.O Box 31 \\ Derby, United Kingdom, DE24 8BJ
}

\begin{abstract}
An optimisation method consisting of the non-dominated sorting genetic algorithm (NSGA-II) and computational fluid dynamics of aero-engine nacelles is outlined. The method is applied to three nacelle lengths to determine the relative performance of different ultra-high bypass ratio engine nacelles. The optimal designs at each nacelle length are optimised for three objective functions: cruise drag, drag rise Mach number and change in spillage drag from mid to end of cruise. The Pareto sets generated from these optimisation computations demonstrate that the design space for short nacelles is much narrower in terms of these performace metrics and there are significant penalties in the off design conditions compared to the longer nacelle. Specifically the minimum spillage drag coefficient attainable, for a nacelle with a drag rise Mach number above 0.87 , was 0.0040 for the shortest nacelle compared to 0.0005 for a nacelle which was $23 \%$ longer.
\end{abstract}

\section{Nomenclature}

$\begin{array}{ll}\mathrm{BPR} & =\text { By-pass ratio } \\ C F D & =\text { Computational fluid dynamics } \\ \mathrm{CST} & =\text { Class shape transformation } \\ \mathrm{FNPR} & =\text { Fan nozzle pressure ratio } \\ \mathrm{FPR} & =\text { Fan pressure ratio } \\ \mathrm{MFCR} & =\text { Mass flow capture ratio } \\ \text { NSGA-II } & =\text { Non-dominated sorting genetic algorithm II } \\ \text { OPR } & =\text { Overall pressure ratio } \\ \text { RANS } & =\text { Reynolds averaged Navier-Stokes } \\ \mathrm{UHBPR} & =\text { Ultra-high bypass ratio } \\ A_{\text {hi }} & \left.=\text { Highlight area [m }{ }^{2}\right] \\ c_{D, \text { cruise }} & =\text { Nacelle drag coefficient in cruise [-] } \\ c_{D, s p i l l} & =\text { Spillage drag coefficient [-] } \\ D_{\text {cruise }} & =\text { Cruise nacelle drag [N] } \\ D_{\text {nac }} & =\text { Nacelle drag [N] } \\ D_{\text {spill }} & =\text { Change in spillage drag [N] } \\ M_{D R} & =\text { Drag rise Mach number [-] } \\ M_{\infty} & =\text { Free stream flight Mach number [-] } \\ M & =\text { Mach number [-] } \\ l_{\text {int }} & =\text { Intake length [m] } \\ l_{f b} & =\text { Nacelle forebody length [m] } \\ & \end{array}$

${ }^{1} \mathrm{PhD}$ Student Researcher, Propulsion Engineering Centre, Cranfield University.

${ }^{2}$ Head of Gas Turbine Technology Group, Propulsion Engineering Centre, Cranfield University, AIAA Member.

${ }^{3}$ MSc Student Researcher, Propulsion Engineering Centre, Cranfield University

${ }^{4}$ Installation Aerodynamics Specialist, Installation Aerodynamics, Rolls Royce plc. 


$\begin{array}{ll}l_{\text {nac }} & =\text { Nacelle length }[\mathrm{m}] \\ r_{\text {fan }} & =\text { Fan radius }[\mathrm{m}] \\ r_{h i} & =\text { Highlight radius }[\mathrm{m}] \\ r_{\text {max }} & =\text { Nacelle maximum radius }[\mathrm{m}] \\ r_{T E} & =\text { Trailing edge radius }[\mathrm{m}] \\ r_{i f} & =\text { Initial forebody radius }[\mathrm{m}] \\ U_{\infty} & =\text { Freestream velocity }\left[\mathrm{ms}^{-1}\right] \\ \beta & =\text { Boat tail angle }\left[{ }^{\circ}\right] \\ \kappa_{T E} & =\text { Trailing edge curvature }\left[\mathrm{m}^{-1}\right] \\ \rho_{\infty} & =\text { Freestream density }\left[\mathrm{kgm}^{-3}\right] \\ \phi_{\text {pre }} & =\text { Pre-entry streamtube rearwards force }[\mathrm{N}] \\ \phi_{\text {nac }} & =\text { Nacelle rearwards force }[\mathrm{N}] \\ \phi_{\text {post }} & =\text { Post-exit streamtube rearwards force }[\mathrm{N}\end{array}$

\section{Introduction}

Gor the past several decades aero-engine manufacturers have followed a trend to increase the by-pass ratio (BPR) to improve the propulsive efficiency by reducing the specific thrust [1]. As this trend continues it is expected to produce an increase in the fan size of turbofan engines enabled by advances in new core architectures and material technology. A typical civil aircraft installation of engines is to locate them under the wings inside a nacelle. The nacelle is optimised to balance the requirement to contribute as litte drag as possible to the overall installation over the full aircraft flight envelope; while also satisfying the requirement to provide an intake and exhaust system with satisfactory flow in terms of compatibility and efficiency. In addition there is also a need to meet noise suppression requirements. A previous study reported an overall nacelle contribution of around $14 \%$ of the drag for a civil twin-engine airliner [2]. A larger fan diameter will result in a larger and heavier nacelle, which is more penalising on the overall aircraft performance. Larger diameters will also result in greater interference between the nacelle and airframe in an underwing installation [3]. It is possible that this increase in weight and drag will overwhelm the cycle efficiency benefits, at an installed powerplant level, gained from the specific thrust reduction. The nacelle design space must therefore be expanded to incorporate shorter and slimmer designs whilst still aerodynamically performing well throughout the flight envelope. The earlier in the design process that such nacelles can be assessed the greater the possibility for optimal design of the overall engine. A previous study [4] which focused on the correct sizing of future engine found that with an increased technology level and BPR increased from 11.5 to 21.5 a reduction in the specific fuel consumption by around $7 \%$ would be expected. However, the overall fuel burn may be increased by adopting too high a BPR and the same study demonstrated that once the installation was considered the optimal engine had a BPR of 14.3. An increase from 14.3 to 21.5 increased the fuel burn by $3.5 \%$ due to added nacelle drag, weight and installation penalty.

It is desirable to have a preliminary assessment of the design space for short and slim nacelles in terms of key performance metrics. One metric of particular importance is the cruise drag $\left(D_{\text {cruise }}\right)$ which should be minimised to limit the overall fuel burn for an aircraft mission. In addition to this however the nacelle must also operate adequately at off design conditions. Two metrics of specific interest are the drag rise Mach number $\left(M_{D R}\right)$ and the spillage drag $\left(D_{\text {spill }}\right)$. The drag rise Mach number is the value at which a rapid increase in drag with freestream Mach number arises and is often defined based on a drag gradient criterion as a function of the freestream Mach number. Though such criteria are not found in the published literature for nacelles, full aircraft drag rise criteria are available. For example, Roskam [5] defines the drag rise Mach number $\left(M_{D R}\right)$ based on a drag gradient (equation (1)). $M_{D R}$ is a key parameter for the assessment of nacelle configurations as a designer will tend to avoid being within 0.02 of the drag rise during cruise [6]. Spillage drag is encountered at certain engine mass flows. The mass flow capture ratio (MFCR) is defined as the ratio of the upstream stream tube area to the nacelle highlight area. Spillage drag occurs has been defined as drag incurred when the value of MFCR decreases below 1; the spillage drag is equivalent to the difference in the suction force from the forebody and the change in momentum of the streamtube [7]. In off-design conditions, such as when the engine is throttled back towards the end of the cruise or in a wind-mill condition, the MFCR is reduced significantly. A typical mid-cruise MFCR for a subsonic aircraft is around MFCR=0.7 [8] which reduces when the engine is throttled back. As a result of this, it is more useful to consider a change in the spillage drag relative to the cruise condition. A critical condition for the engine nacelle occurs when the engine is not operable and the fan freely rotates, this is called the wind mill and is typically considered for the take off condition. Under the wind-milling condition the MFCR can be reduced to MFCR=0.3 [9]. This large reduction in MFCR can result in very high values of spillage drag which creates a yaw moment for 
the aircraft and to balance this yaw moment the vertical stabiliser must be appropriately sized. One study [4] identified that an increase in fan diameter from $2.84 \mathrm{~m}$ to $3.78 \mathrm{~m}$ on the B777-200ER at a Mach number of $M_{\infty}=0.2$ , similar to a take off condition, resulted in an increase of wind-mill drag of over $150 \%$ which required an increase in the tail size of $15 \%$.

$$
\left.\frac{\partial c_{D}}{\partial M_{\infty}}\right|_{\mathrm{M}=\mathrm{M}_{\mathrm{DR}}}=0.1
$$

To address the need for appropriate nacelle design, previous efforts have been made to optimise the aero-lines of the external and internal cowl [10] [11] [12]. However, a major hurdle in optimising the design is that many objectives must be satisfied concurrently so high fidelity models are too expensive to use. Therefore, work has often been focused on utilising lower order methods or surrogate modelling to map out a design space [12]. Whilst these methods can offer great reductions in computational expense they rely on interpolation between data and are therefore at risk of providing false optima. As computational power becomes more readily available it has become possible to move to higher fidelity models for use in optimisation and design. Optimisations including full computational fluid dynamics (CFD) analyses as the evaluations have been undertaken previously [11] and demonstrate that detailed analysis of the aerodynamics are then possible. Previous work has also focussed on determining optimal parametric models to aid in producing better performing aerodynamic designs [10]. This work demonstrated that for a nacelle optimisation with two objective functions, inner and outer peak Mach numbers, class shape transformation (CST) curves gave more optimal designs than B-splines or superellipse polynomials [10].

The aim of this paper is to demonstrate an optimisation method applied to nacelle design using CFD simulations. Multi-objective optimisation is used to concurrently optimise three aerodynamic design parameters to produce Pareto fronts for a given nacelle length. The proposed method allows a trade-off to be made between the three aerodynamic parameters and the difference in performance metrics which can be achieved at different nacelle lengths. This enables a direct analysis of the viability of short and slim nacelle designs for future aero-engines in terms of both their performance metrics and the aerodynamics.

\section{Methods and scope}

To explore the design space of shorter and slimmer nacelle designs, an optimisation approach has been coupled with computational fluid dynamics (CFD) computations. The optimisation has been achieved using NSGA-II [13] which is an evolutionary algorithm. NSGA-II was chosen for its elitism which maintains good designs throughout later generations and due to its convergence speed. The routine follows a loop (Figure 1) which progresses through a set number of generations of vectors that are converted into nacelle parameters and used to create geometries, computational meshes and CFD boundary conditions. The routine uses blend crossover and Gaussian mutation operators on the design variables to provide variance from antecedent nacelle designs [14].

In this study, a nacelle/intake/nozzle geometry is defined as a series of CST curves. These consist of a class function which is weighted by a shape function of superimposed Bernstein polynomials [15]. The method was expanded to allow intuitive (iCST) design variables to be used in place of direct specification of the coefficients of the Bernstein polynomials, allowing designers to specify more typical geometric constraints such as curvature at a position [16]. A parameterisation based on iCST curves was established which used these intuitive nacelle parameters to define a nacelle geometry (Figure 2). These intuitive parameters include the highlight radius $\left(\mathrm{r}_{\mathrm{hi}}\right)$, which directly determines the highlight area and thus the MFCR for a given freestream aerodynamic condition and intake mass flow. The maximum radius $\left(r_{\max }\right)$ determines the frontal facing area of the nacelle. The forebody length $\left(\mathrm{l}_{\mathrm{fb}}\right)$ and the maximum radius $\left(\mathrm{r}_{\max }\right)$ are chosen together to allow a favourable curvature distribution and thus $\mathrm{c}_{\mathrm{p}}$ distribution over the nacelle forebody. This requires a certain amount of initial forebody curvature, $\frac{1}{r_{i f}}$, to control the acceleration over the forebody as much as possible. Some older nacelles have a cylindrical centrebody of length which allows the forebody and afterbody geometries to be separated and reduces the interactions between the aerodynamics of these two sections. This however is less likely to be desirable in larger nacelle designs owing to the additional weight. The remainder of the nacelle length $\left(l_{n a c}\right)$ gives the distance from the maximum diameter to the trailing edge and must be of sufficient length to allow the gradient to transition smoothly from zero at the maximum radius to the required boattail angle $(\beta)$. The overall length $\left(l_{n a c}\right)$ of the nacelle is key in order to reduce skin friction drag and weight therefore should be minimised as far as possible particularly if a greater fan diameter is used. The trailing edge radius $\left(\mathrm{r}_{\mathrm{TE}}\right)$ is dictated by the requirements of the engine design to achieve the cycle design point and optimised for efficiency and operability. Two iCSTs were used to describe the nacelle cowl to allow greater control 
within the optimisation. This 'hybrid-iCST' allows the forebody and afterbody to differ in curvature distributions in comparison to that created by a single iCST used to define the whole nacelle aeroline. The intersection of the two iCSTs is the maximum radius of the nacelle curve. At this point, the first, second and third derivatives of the iCST are matched between the two curves to ensure no curvature discontinuity which would create undesirable aerodynamic effects. At the intersections between the other curves similar limitations were put in place. At the highlight point, the intake and forebody nacelle iCST meet, with an infinite value of gradient and a variable curvature which implicitly prescribes the second derivative. The optimisation process was carried out with five of the nacelle parameters $\left[l_{f b}, r_{\text {max }}, r_{i f}, \beta, \kappa_{T E}\right]$, treated non-dimensionally as degrees of freedom in the optimisation and the remainder $\left[l_{n a c}, r_{h i}, r_{T E}, l_{\text {int }}\right]$ being fixed to a specific value.

To provide representative boundary conditions for the numerical simulations at the nozzle inlets two engine cycles were developed. The cycles, developed in a zero dimensional cycle modelling tool [17] [18], both represented ultra-high bypass ratio (UHBPR) turbofan engines. The engine cycles were designed for a cruise altitude of $10,668 \mathrm{~m}$ and one had a 3 shaft architecture whilst the other had a 2 shaft architecture with a gearbox which is more representative of a future UHBPR [19]. To represent a more challenging design point, the 2 shaft architecture has a higher cruise Mach number and lower mass flow capture ratio (MFCR). The cycles, summarised in Table 1, were modelled at two power settings to ensure the efflux could be modelled proportionately to the airflow through the engine when MFCR was reduced. Two baseline nacelle designs were developed for these cycles. For the three shaft cycle, a long nacelle was produced with a non-dimensional length of $\frac{\mathrm{l}_{\text {nac }}}{\mathrm{r}_{\mathrm{hi}}}=4.3$ and for the two shaft cycle a more aggressive length of $\frac{l_{\text {nac }}}{r_{h i}}=3.1$ to represent an increase in nacelle technology.

Table 1 - Summary of engine cycle parameters at cruise point

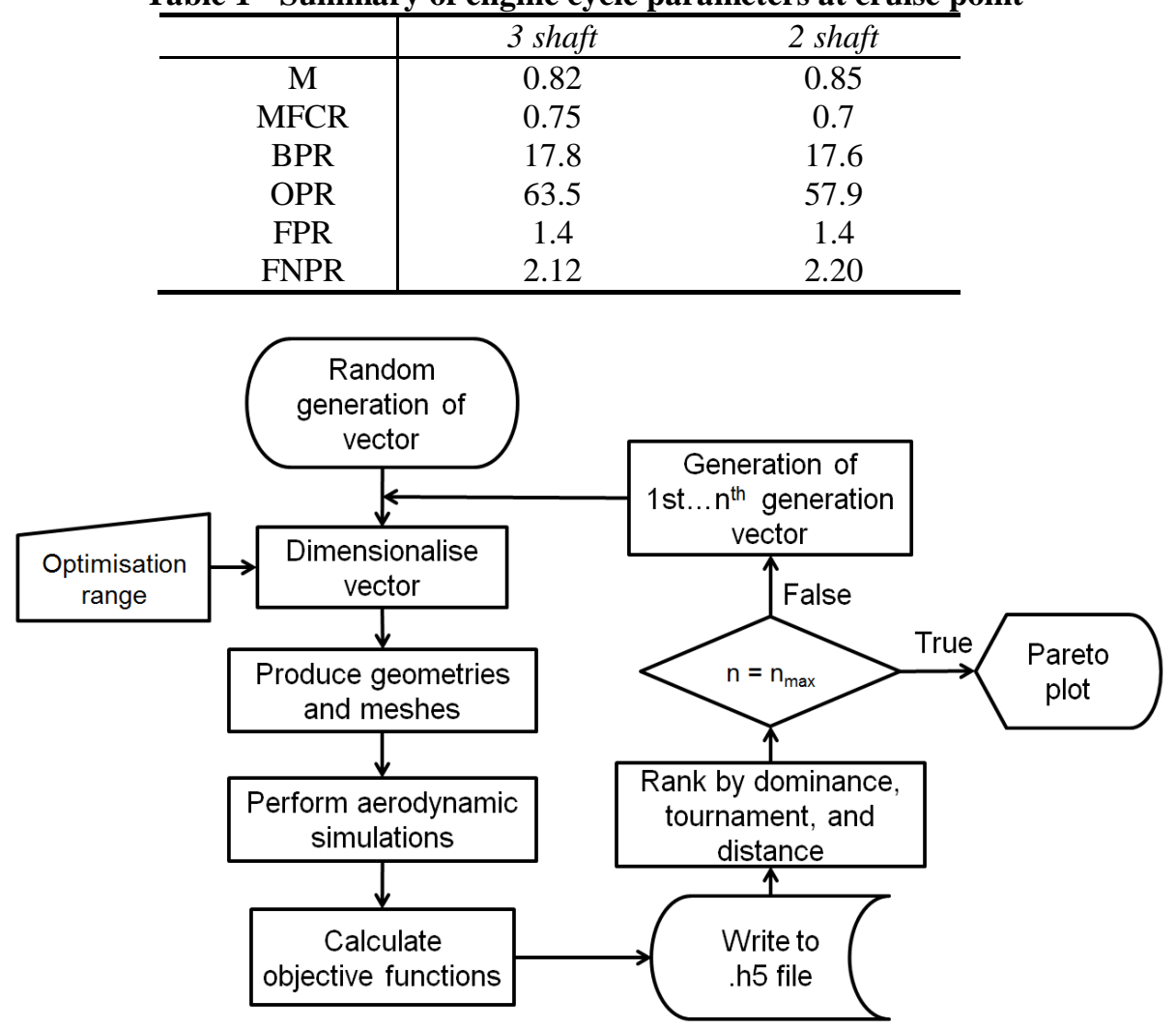

Figure 1. Optimisation routine outlined as a flow chart 


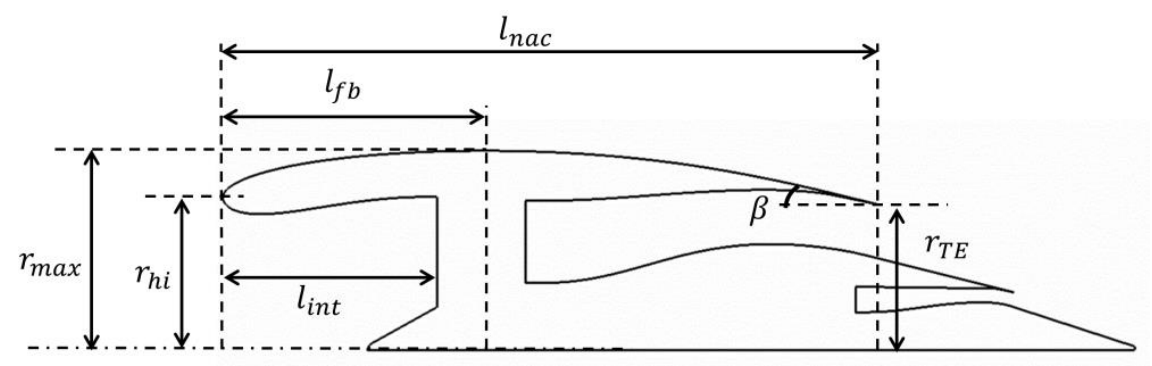

Figure 2. iCST Nacelle parameterisation

The steady Reynolds Averaged Navier Stokes (RANS) equations were solved using double precision for all cases. The analyses of these nacelle designs have been carried out using a steady RANS CFD approach [20]. An implicit density-based solver was used with second order discretisation and a Green-Gauss node based scheme. The turbulence model was the two equation $\mathrm{k}-\omega$ SST model which was chosen for the improved boundary layer modelling over the standard $\mathrm{k}-\varepsilon$ model. CFD analyses were considered iteratively converged when normalised residual values reduced below $10^{-5}$ and fluctuations in the mass flow measured at the engine inlet were below $0.01 \%$. The 2D axisymmetric computations used a semi-circular domain generated around the geometries with a radius of $80 r_{\max }$ set in accordance with a domain sensitivity study. A multi-block structured mesh was produced for each of approximately 70000 cells. A mesh independence study was carried out with three grids of 35000, 70000 and 140000 cells. In accordance with the suggested reporting format set out by Roache [21] it was found that the 70000 cell grid led to spatial discretisation errors less than $1 \%$ in drag.

To determine the performance of the engine nacelles in flight from the CFD results, the modified nearfield method [22] was used. The nacelle drag $\left(D_{\text {nac }}\right)$ [23] includes contributions from pre-entry $\left(\phi_{\text {pre }}\right)$ and post exit forces $\left(\phi_{\text {post }}\right)$ as well as pressure and skin friction forces acting on the external surface of the nacelle. The modified near field method allows the pre-entry and nacelle forces to be computed concurrently so that the highlight stagnation point does not need to be extracted. The post-exit streamtube was extracted from the trailing edge of the nacelle and the pressure forces integrated along it to determine the post-exit axial force. The first objective function used for the optimisation was the mid cruise drag incurred by the nacelle $\left(D_{\text {cruise }}\right)$, measured at $M_{\infty}=0.82$ and MFCR $=0.75$ for the 3 shaft engine, and $M_{\infty}=0.85$, and MFCR $=0.7$ for the 2 shaft engine. In addition to the nacelle drag, two additional terms are considered. The first is the drag rise Mach number which is defined by a gradient criterion, as given by Shevell [24], (equation (1)). The second is the spillage drag (equation (2)) between the cruise condition and a MFCR representative of end of cruise while at constant Mach number. Nacelle drag and spillage drag are reported throughout this paper as a non-dimensional coefficient (equations (3)(4)) related to the area of the highlight because the maximum area is a variable in the optimisation.

$$
\begin{gathered}
D_{\text {spill }}=D_{M F C R=0.65}-D_{\text {cruise }} \\
c_{D, \text { cruise }}=D_{\text {cruise }} / 0.5 \rho_{\infty} U_{\infty}^{2} A_{h i} \\
c_{D, \text { spill }}=D_{\text {spill }} / 0.5 \rho_{\infty} U_{\infty}^{2} A_{h i}
\end{gathered}
$$

\section{Results and analysis}

\section{A. Optimisation of conventional length nacelle}

To demonstrate the applicability of the genetic algorithm, an optimisation of the three objective functions was carried out for a non-dimensional nacelle length of $\frac{l_{n a c}}{r_{h i}}=4.3$. This is a particularly long nacelle and was expected to represent a benign and 'conventional' aerodynamic performance. Boundary conditions for the nozzle inputs in the CFD analyses came from the cycle model for the 3 shaft cycle (Table 1). The bounds used for the degrees of freedom, summarised in Table 2, were chosen to represent a conventional range of nacelle parameters whilst avoiding concave regions in the nacelle. The optimisation began with a random generation of 4005 -dimensional vectors, which were converted to a generation of nacelle designs through the parameterisation (Figure 3a). Over 30 generations, the population was adapted through mutation and crossover, maintaining the individuals with the best fitness and using these to create additional designs (Figure 3b, c, and d). Through the 30 generations in the 
optimisation, 1560 different nacelle designs were evaluated, which consisted of 21,840 individual aerodynamic analyses.

To determine the convergence of the optimisation on a Pareto set, the hypervolume of the Pareto set was calculated at each generation. Hypervolume [25] is defined as an m-dimensional volume in the objective space which is measured relative to an arbitrary reference value. This metric gives an indication as to the convergence onto a Pareto set and also the overall spread of the set. Two problems arise with the definition of hypervolume for this optimisation. Firstly, since one objective is to be maximised and two minimised the optimal hypervolume would be decreasing in one dimension whilst increasing in the other two. To address this, when hypervolumes were calculated values of $1-M_{D R}$ were used. Secondly, the scales of the objective functions being dealt with are vastly different, with the cruise drag being around an order of magnitude smaller than the drag rise Mach number and the spillage drag being another order of magnitude smaller. To make the hypervolume calculation represent the three objectives more evenly, they have been divided by the maximum values found in each objective throughout the optimisation. A reference point was used of $(1,1,1)$. The hypervolume for the optimisations have all been computed using open source code [26]. Over the final 5 generations, there was a $0.2 \%$ variation in the hypervolume which suggested the algorithm was converged. The improvement from the random sample resulted in a mean value improvement of $\Delta M_{D R}=0.0034$, from $M_{D R}=0.8682$ to $M_{D R}=0.8717$. The mean value of drag $\left(c_{D \text { cruise }}\right)$ reduced by $\Delta c_{D \text { cruise }}=$ 0.0010 from $c_{\text {Dcruise }}=0.0350$ to $c_{\text {Dcruise }}=0.0339$, whilst the mean spillage $\left(c_{\text {Dspill }}\right)$ remained unchanged. At such nacelle lengths however the spillage for most of the designs is of a very low value.

This process ultimately produced a Pareto front of designs in which each is non-dominated (Figure 4). Results from this optimisation (Figure 4) demonstrated a range of available cruise drag coefficient values between 0.031 and 0.048. At this length, a drag rise Mach number of 0.894 can be achieved. At high drag rise Mach numbers there is an increase in the spillage drag which demonstrates the compromise between these two performance metrics.

Table 2. Bounds of degrees of freedom in optimisation

\begin{tabular}{c|ll}
\hline Parameter & Lower bound & Upper bound \\
\hline$l_{f b} / r_{h i}$ & 0.36 & 0.48 \\
$r_{\max } / r_{h i}$ & 1.2 & 1.35 \\
$\beta$ & 11 & 14 \\
$\kappa_{T E}$ & 0 & 1 \\
$r_{i f} l_{f b} /\left(r_{\max }-r_{h i}\right)^{2}$ & 0.8 & 1.2 \\
\hline
\end{tabular}



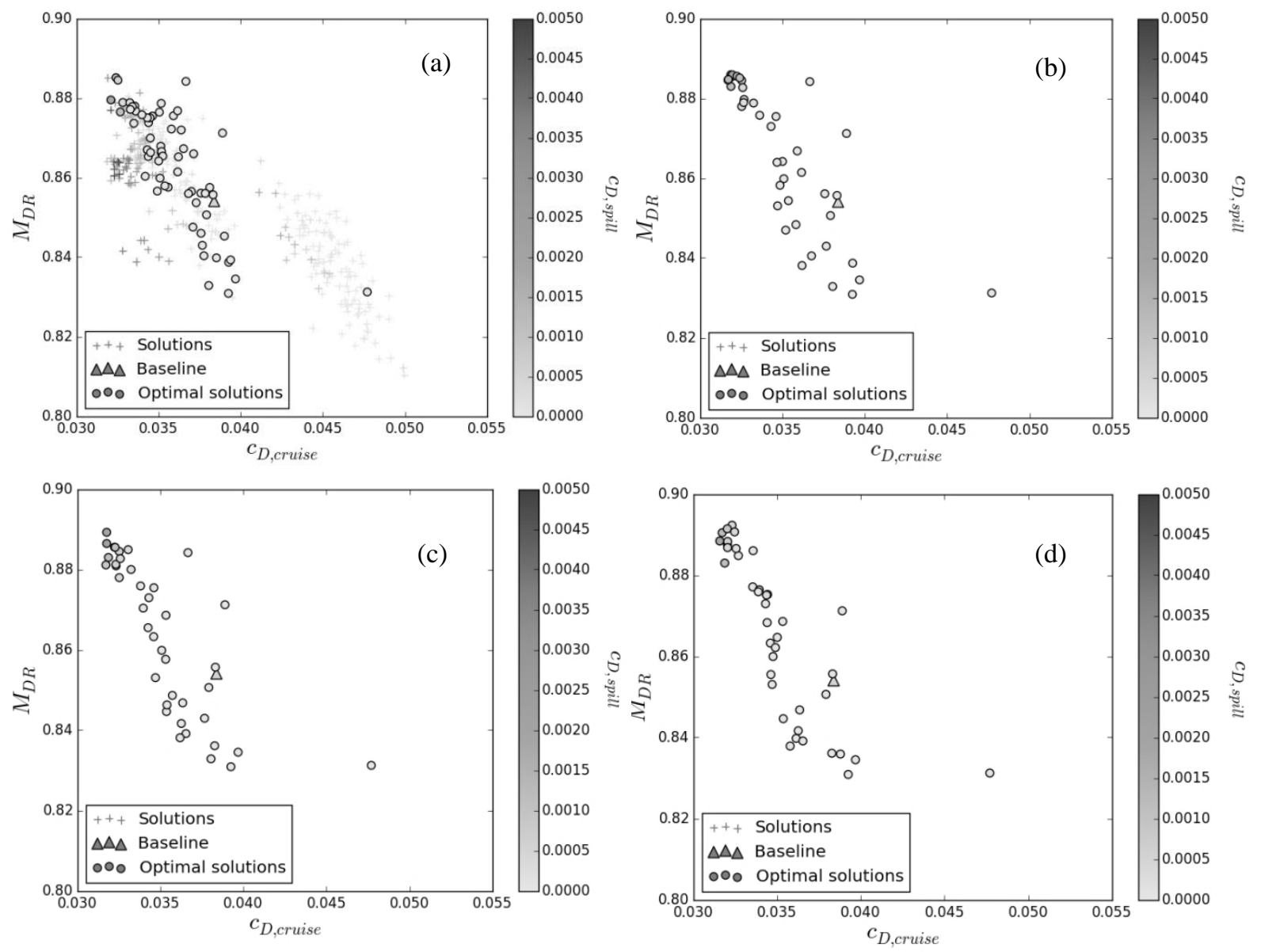

Figure 3. Population in objective space for (a) generation 0 (b) generation 5 (c) generation 10 and (d) generation 20

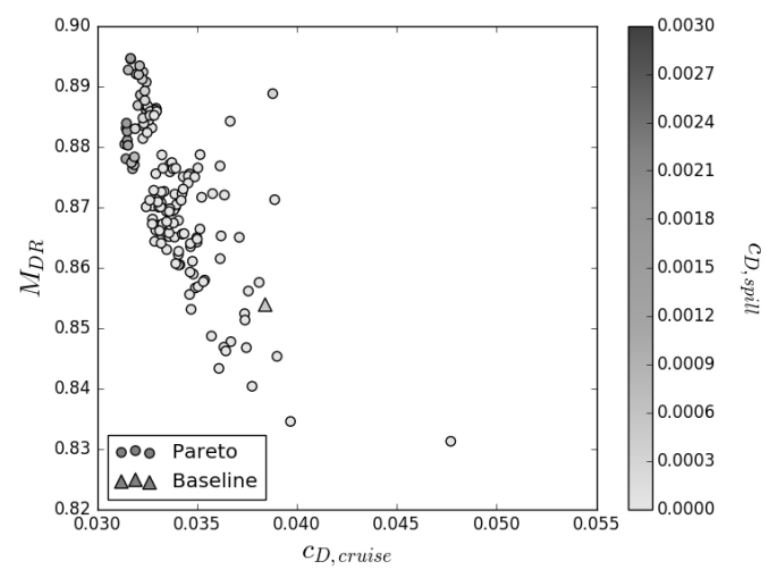

Figure 4. Final Pareto optimal set for nacelle length of $\frac{l_{n a c}}{r_{h i}}=4.3$ for the 3 shaft engine determined by NSGA-II algorithm in objective space 
The isentropic Mach number distributions of the nacelles produced from optimisation of the three objective functions show that the designs exhibit typical nacelle aerodynamics (Figure 5). High drag rise Mach numbers can be achieved without a large increase in the peak Mach number at the cruise MFCR. However, the decrease in MFCR which arises at the off-design condition, creates a more pronounced acceleration over the lip, which is the cause of the increase in spillage drag at high drag rise Mach numbers.
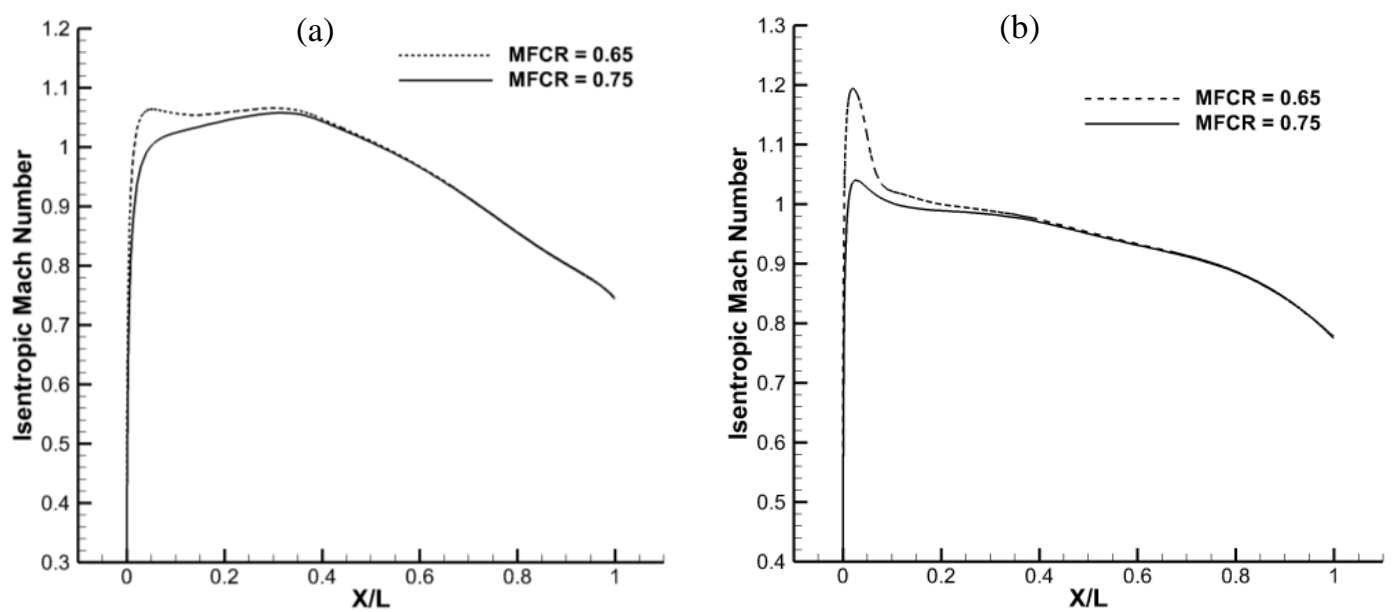

Figure 5. Nacelle isentropic Mach number distributions for optimal designs for the nacelle length $\frac{l_{\text {nac }}}{r_{h i}}=4.3$

with (a) $M_{D R}=0.84$ (b) $M_{D R}=0.88$

\section{B. Optimisation of advanced nacelles}

To understand the nacelle design space available for the 2 shaft engine cycle, optimisations were carried out for the three objective functions at two nacelle lengths. An optimisation was carried out for a non-dimensional nacelle length of $\frac{l_{h a c}}{r_{h i}}=3.8$. The bounds used for this optimisation, summarised in Table 3, were chosen to cover a wide range of the available design space and expand beyond the conventional design parameters.

The hypervolume was again calculated for the Pareto set at each generation to determine the level of convergence and the improvement from the optimisation process. Over the final 5 generations there was less than a $1 \%$ increase in hypervolume. The improvement from the random sample resulted in a $\Delta M_{D R}=0.0044$ higher mean value of $M_{D R}$ which increased from $M_{D R}=0.8672$ to $M_{D R}=0.8716$. The mean value of drag $\left(c_{D c r u i s e}\right)$ reduced by $\Delta c_{\text {Dcruise }}=0.0009$ from $c_{\text {Dcruise }}=0.0313$ to $c_{\text {Dcruise }}=0.0304$, however the mean spillage $\left(c_{\text {Dspill }}\right)$ increased from $c_{D \text { spill }}=0.0027$ to $c_{\text {Dspill }}=0.0040$ as the optimisation populated the objective space with high drag rise designs. The minimum spillage drag which met the criterion of $M_{D R}=0.87$ decreased by $\Delta c_{D s p i l l}=0.0011$ from $c_{\text {Dspill }}=0.0016$ to $c_{\text {Dspill }}=0.0005$.

Since the engine design is intended to cruise at a flight $M_{\infty}=0.85$, it is expected that the drag rise Mach number be at least 0.87 [6]. This requirement is caused by the typical increase in local velocity caused by an underwing installation. A constraint was therefore applied of $M_{D R} \geq 0.85$ to resolve this region of the design space. The results of this optimisation demonstrate that the higher drag rise Mach numbers are achieved at the expense of the spillage drag performance. Above the required $M_{D R}$ of 0.87 , the spillage drag is consistently above $5 \%$ of the cruise drag. The lower values are achieved at the expense of higher mid cruise drag (Figure 6) however this is still more penalising than the spillage of the longer nacelle $\left(\frac{l_{n a c}}{r_{h i}}=4.3\right)$ which was typically less than $2 \%$ of the cruise drag (Figure 4). 
Table 3. Bounds of degrees of freedom in optimisation

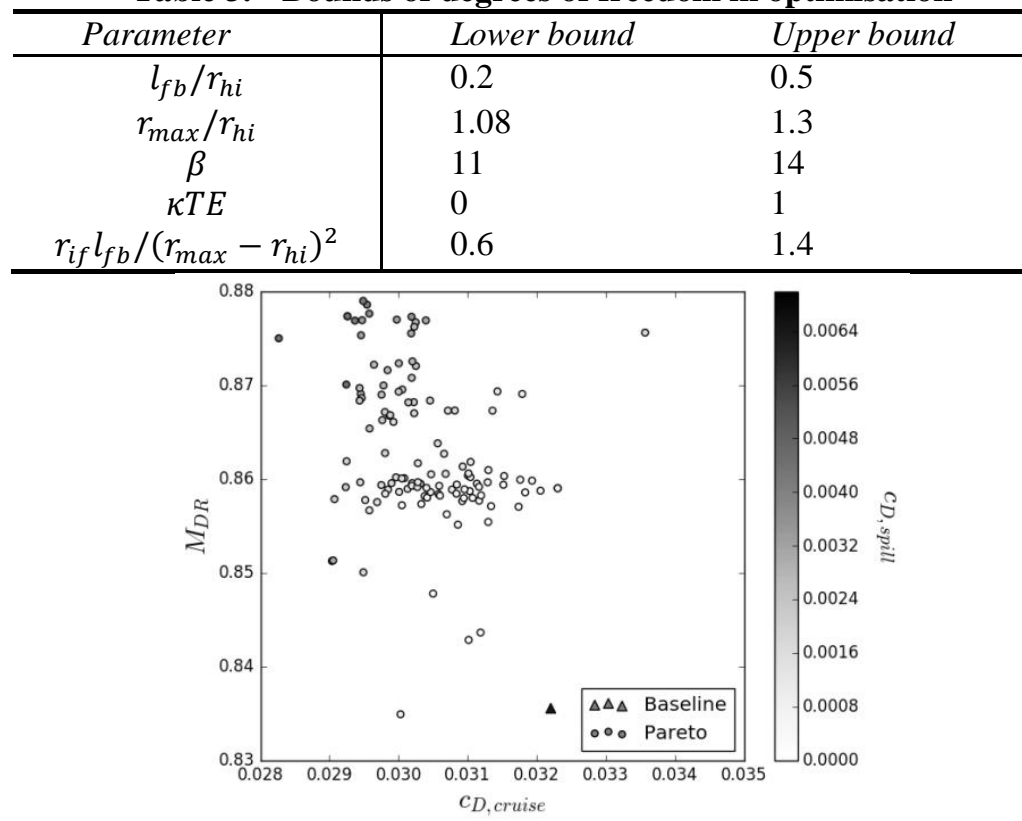

Figure 6. Final Pareto optimal set for nacelle length of $l_{n a c} / r_{h i}=3.8$ determined by NSGA-II algorithm in objective space

To assess a very aggressive nacelle length, an optimisation was carried out for a non-dimensional nacelle length of $\mathrm{l}_{\text {nac }} / \mathrm{r}_{\mathrm{hi}}=3.1$. The same non-dimensional bounds were used on the 5 degrees of freedom as were used for the optimisation of the longer nacelle (Table 3). Again the convergence was determined based on the hypervolume as a function of the generation number. Over the final 5 generations there was less than a $0.1 \%$ increase in hypervolume. The improvement from the random sample resulted in a $\Delta M_{D R}=0.0046$ higher mean value of $M_{D R}$ with an increase from $M_{D R}=0.8667$ to $M_{D R}=0.8805$. The mean value of drag $\left(c_{\text {Dcruise }}\right)$ reduced by $\Delta c_{D \text { cruise }}=0.0028$ from $c_{\text {Dcruise }}=0.0311$ to $c_{\text {Dcruise }}=0.0283$, whilst the mean spillage $\left(c_{\text {Dspill }}\right)$ decreased by $\Delta c_{\text {Dspill }}=0.0048$ from $c_{D \text { spill }}=0.0101$ to $c_{D \text { spill }}=0.0053$. No individuals in the initial random design space exceeded a $M_{D R}=0.87$. However after the optimisation $34 \%$ of the Pareto set had a $M_{D R} \geq 0.87$ with a minimum spillage, for a design which meets the criterion, being $c_{\text {Dspill }}=0.0040$. The results from the optimisation are shown after 30 generations (Figure 7). It is clear from these results that the optimal designs at this shorter nacelle length $\left(\mathrm{l}_{\text {nac }} / \mathrm{r}_{\mathrm{hi}}=3.1\right)$ allow for around $10 \%$ lower nacelle drag than at the longer length $\left(\mathrm{l}_{\mathrm{nac}} / \mathrm{r}_{\mathrm{hi}}=3.8\right)$. This is to be expected since there is less wetted area for a shorter nacelle and the skin friction drag of the nacelle typically accounts for 50-60\% of the nacelle drag. However, these optimal designs also demonstrate that reaching the higher values of drag rise Mach numbers is more penalising to the spillage drag at lower MFCRs. This also resulted in less desirable pressure distributions on the nacelle surface which produced this additional wave drag at off design conditions. This highlights the increased difficulty in designing a nacelle of shorter length as the spillage drag $\left(c_{D, \text { spill }}\right)$ is around twice as large at $\mathrm{M}_{\mathrm{DR}}=0.87$ for the shorter length $\left(\mathrm{l}_{\mathrm{nac}} / \mathrm{r}_{\mathrm{hi}}=3.1\right)$ in comparison to the longer nacelle $\left(\mathrm{l}_{\text {nac }} / \mathrm{r}_{\mathrm{hi}}=\right.$ 3.8). 


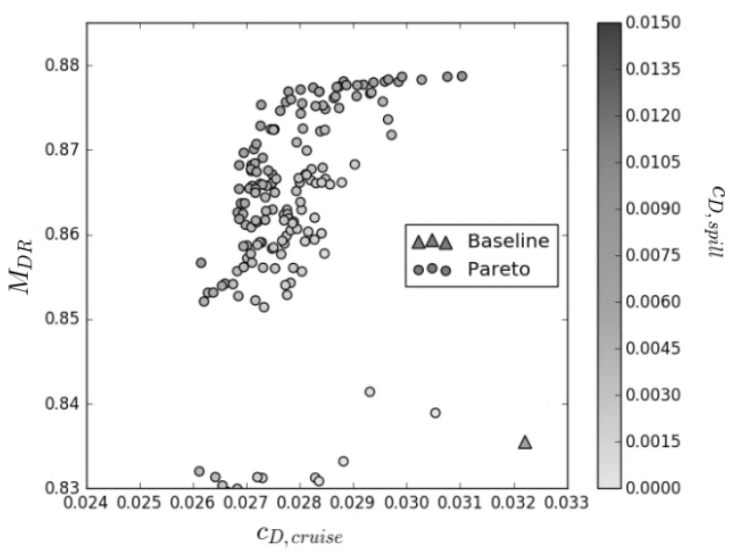

Figure 7. Final Pareto optimal set for nacelle length of $l_{n a c} / r_{h i}=3.1$ determined by NSGA-II algorithm

\section{in objective space}

\section{Relative importance of design space variables}

To assess the most important variables in the design of nacelles, the final Pareto set generated from the optimisation was examined (Figure 8). A comparison between the final Pareto set of the $\mathrm{l}_{\text {nac }} / \mathrm{r}_{\mathrm{hi}}=3.8$ optimisation and the $\mathrm{l}_{\text {nac }} / \mathrm{r}_{\mathrm{hi}}=3.1$ optimisation show some similarities. For instance both show a clear trend in the first three design variables $\left(l_{f b} / r_{h i}, r_{\max } / r_{h i}\right.$ and $\left.r_{i f} l_{f b} /\left(r_{\max }-r_{h i}\right)^{2}\right)$, where these variables have all converged to a narrow region in the lower half of the design space aside for some outliers. The final two geometric variables $\left(\kappa_{T E}, \beta\right)$ seem to have less influence on the outcome since they are more scattered in the design space, though in general lower boattail angles seem to be preferential. The shorter nacelle optimisation generally converged to a thinner design and had a lower initial forebody radius. The lower the initial forebody radius of a nacelle the sharper the profile will be and the greater the curvature around the lip. This has a negative influence on the spillage characteristics as noted in the position of the optimal spillage design in both nacelle lengths (Figure 8). However by increasing the curvature at the forebody a slimmer nacelle can be achieved without as much rate of change of curvature over the forebody. In both optimisation cases, the design which achieved the highest drag rise also had the highest spillage drag. Similarly the design with the lowest spillage generally had a low drag rise Mach number. This emphasises the compromise needed between the two objectives and the solid line demonstrates that the minimum spillage attainable with a MDR $>0.87$ increases from 0.0005 to 0.0040 by choosing a shorter length nacelle. 


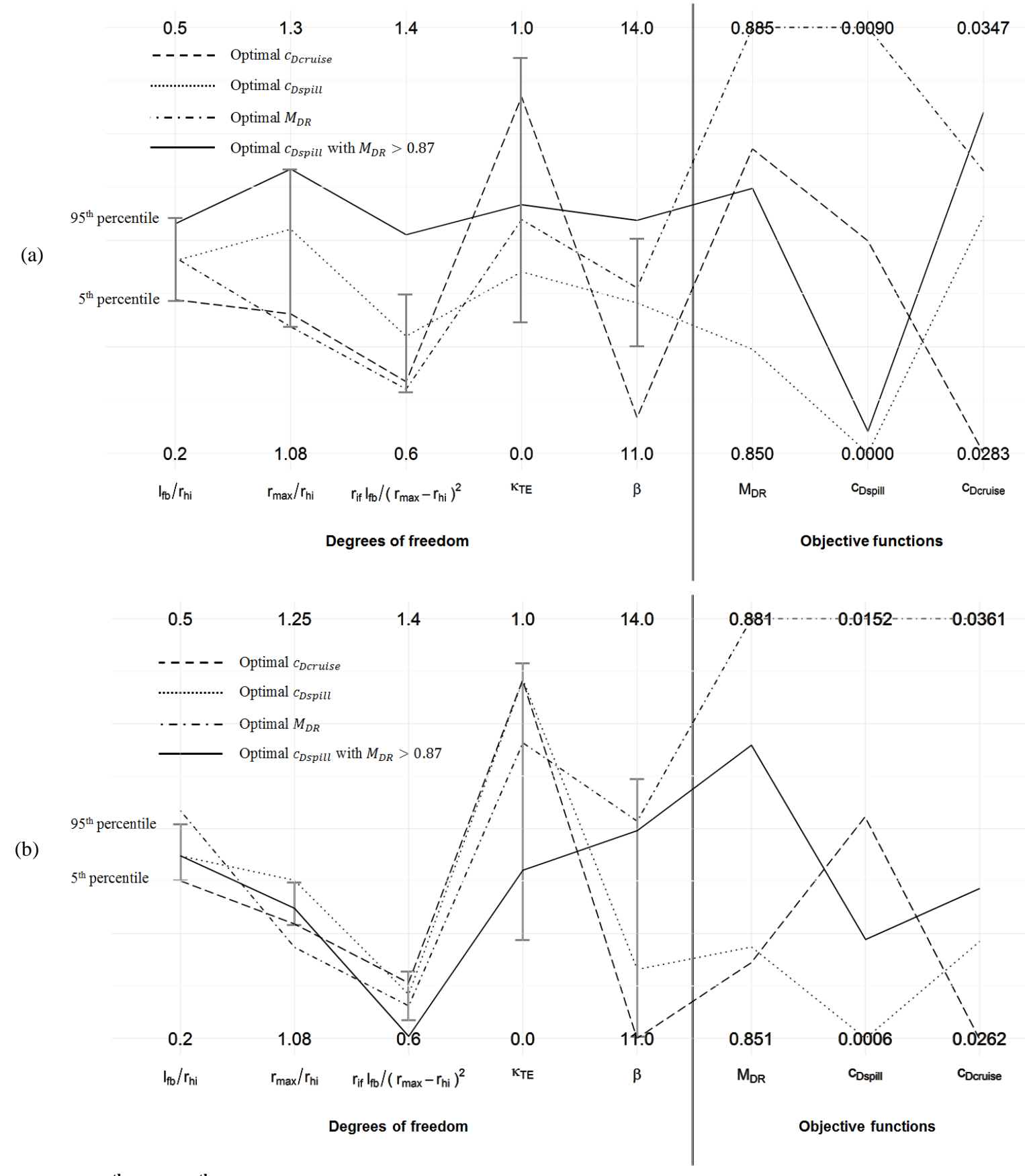

Figure $85^{\text {th }}$ and $95^{\text {th }}$ percentiles of the final Pareto set from the optimisation of nacelle as a function of the geometrical variables, and objective functions with four examples specifically plotted for nacelles lengths of (a) $l_{n a c} / r_{h i}=3.8$ and (b) $l_{n a c} / r_{h i}=3.1$ 


\section{Effect of thickness ratio on objectives}

The thickness ratio $\left(r_{\max } / r_{h i}\right.$, Figure 2$)$ is an important parameter considering the requirement of developing overall slimmer nacelles. It is therefore a notable result that the optimisation for the shorter nacelle $\left(\mathrm{l}_{\text {nac }} / \mathrm{r}_{\mathrm{hi}}=3.1\right)$ produced optimal designs with exclusively lower thickness ratios than the optimisation of the longer nacelle $\left(l_{\text {nac }} / \mathrm{r}_{\mathrm{hi}}=3.8\right)$ (Figure 9). This was predominately driven by the requirement of nacelles with a high drag rise Mach number and with a shorter nacelle higher rates of curvature were needed to achieve this and therefore less thickness could be attained. This results in an average $\frac{r_{\max }}{r_{h i}}=1.13$ for the shorter nacelle $\left(\mathrm{l}_{\mathrm{nac}} / \mathrm{r}_{\mathrm{hi}}=3.1\right)$ and $\frac{r_{\max }}{r_{h i}}=1.18$ for the longer $\left(\mathrm{l}_{\mathrm{nac}} / \mathrm{r}_{\mathrm{hi}}=3.8\right)$. These slimmer designs however come at the expense of the off-design performance such as spillage drag. However, in general the reduction of the thickness and length achieved would allow a volume reduction by using a shorter and slimmer nacelle. With a lower drag and lower weight, the shorter nacelles could allow significant fuel burn reduction if the off-design performance can be improved. The increased spillage is also partially offset by the lower cruise drag present in the shorter nacelle. Slimmer nacelles would also allow an UHBPR engine to be more easily integrated under a wing due to improved ground clearance.
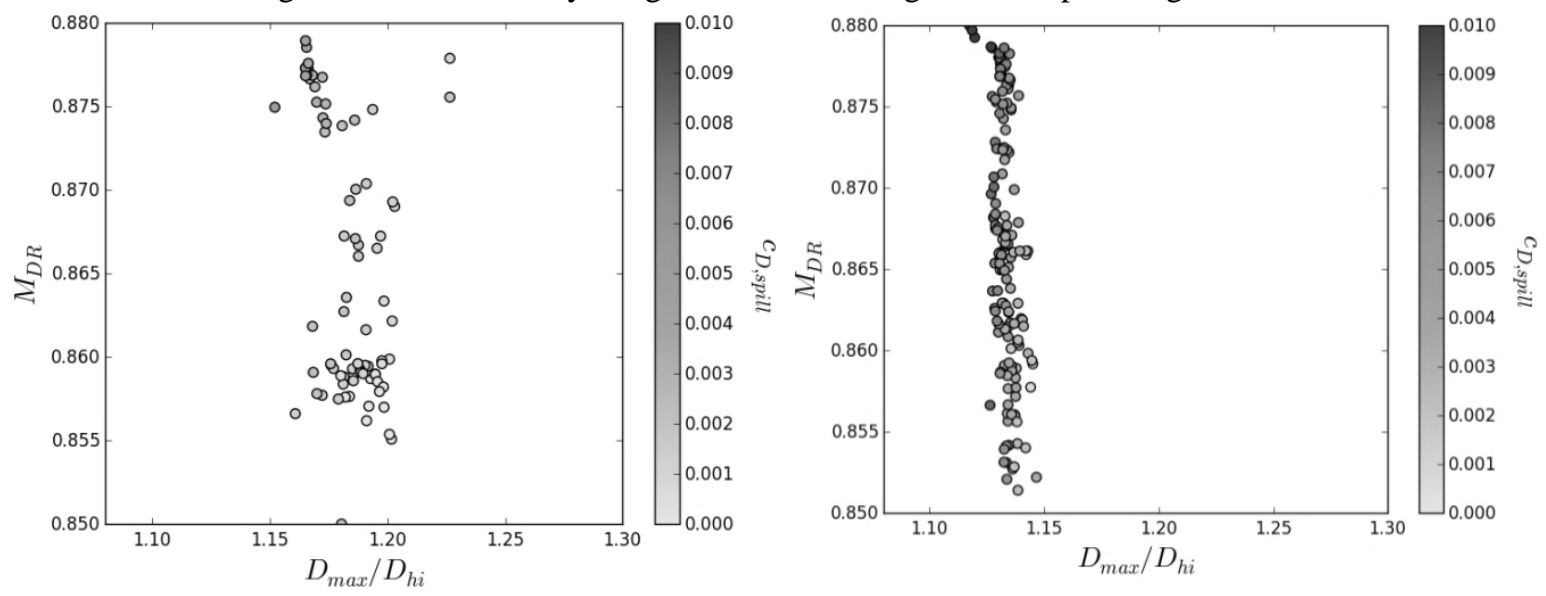

Figure 9 Optimal set of designs for (a) Nacelle length $l_{n a c} / r_{h i}=3.8$ and (b) Nacelle length $l_{n a c} / r_{h i}=$

\section{Conclusions}

An evolutionary algorithm has been coupled with CFD simulations to optimise nacelle designs for three performance metrics simultaneously. Two example engine cycles have been produced representing a 3 shaft and a 2 shaft architecture. Nacelles have been developed for these engine models at various nacelle lengths. The conventional 3 shaft engine nacelle was optimised for cruise drag, drag rise Mach number and spillage drag at $l_{n a c} / r_{h i}=4.3$. The results demonstrated that a large improvement over the baseline was possible and drag rise Mach numbers up to 0.894 achievable. However the spillage drag was seen to increase at the highest drag rise Mach numbers. This trend was more prevalent in the optimisation of the shorter nacelles for the 2 shaft engine. For the optimisation of the 2 shaft engine, two nacelle lengths have been considered, $l_{n a c} / r_{h i}=3.8$ and $l_{n a c} / r_{h i}=3.1$. Both of these lengths demonstrated a lower achievable drag rise Mach number and a greater spillage drag penalty at higher drag rise Mach numbers. The optimisation of shortest nacelle studied also converged to the slimmest designs which suggests there may be an overall benefit in weight and the drag integrated along an typical flight plan if the wave drag can be reduced.

\section{Acknowledgments}

The lead author of this paper was partially supported by a grant from the Engineering and Physical Sciences Research Council. The authors would like to thank Rolls Royce plc. for their support in this project. 


\section{References}

1. Parker R., "From blue skies to green skies: engine technology to reduce the climate-change impacts of aviation", Technology Analysis \& Strategic Management, Vol. 21, No. 1, 2009, pp. 61-78

2. Hoheisel H., "Aerodynamic Aspects of Engine-Aircraft Integration of Transport Aircraft", Aerospace Science and Technology, Vol. 1, No. 7, 1997, 475-487

3. Stankowski T. P., MacManus D. G., Sheaf C. T., and Grech N., "Aerodynamic interference for aero-engine installations", 54 ${ }^{\text {th }}$ AIAA Aerospace Sciences Meeting, AIAA SciTech, Washington, AIAA 2016-0766, DC, 2016

4. Daggett D. L., Brown S. T., and Kawai R. T., "Ultra-Efficient Engine Diameter Study”, NASA, CR-2003-212309, 2003

5. Roskam J., and Lan C.-T.E., Airplane Aerodynamics and Performance, $3^{\text {rd }}$ ed., DARcorporation, Lawrence, KA, 1997, p. 141

6. Borradaile J. A., "Towards the optimum ducted UHBR engine", AIAA/AHS/ASME/ASEE 24 $4^{\text {th }}$ Joint Propulsion Conference, AIAA-88-2954, Washington, DC, 1988

7. Langley M., "The Design of Axisymmetric Cowls for Podded Nacelles for High By-Pass Ratio Turbofan Engines", Aircraft Research Association Limited, Reports and Memoranda no. 3846, Bedford, England, May 1979

8. Farokhi S., Aircraft Propulsion, $1^{\text {st }}$ ed., Wiley, Chichester, England, 2009, p.241

9. Obert E., Aerodynamic Design of Transport Aircraft, $1^{\text {st }}$ ed., IOS Press, Amsterdam, 2009, pp. 491-492

10. Albert M., and Bestle D., "Aerodynamic Design Optimization of Nacelle and Intake", Proceedings of ASME 2013 Turbo Expo: Turbine Technical Conference and Exposition, GT2013-94857, New York, 2013

11. Toubin H., Salah El Din I., and Meheut M., "Multipoint Aerodynamic High Fidelity Shape Optimization of an Isolated Engine Nacelle", 52 ${ }^{\text {nd }}$ AIAA Aerospace Sciences Meeting, AIAA SciTech, AIAA 2014-0903, Washington, DC, 2014

12. Song W., and Keane A., "Surrogate-Based Aerodynamic Shape Optimization of a Civil Aircraft Engine Nacelle", AIAA Journal, Vol. 45, No. 10, 2007, pp. 2565-2574

13. Deb K., Pratap A., Agarwal S., and Meyarivan T., "A Fast and Elitist Multiobjective Genetic Algorithm: NSGA-II", IEEE Transactions on Evolutionary Computation, Vol. 6, No. 2, 2002, pp. 182-197

14. Deb K., Multi-Objective Optimization Using Evolutionary Algorithms, $1^{\text {st }}$ ed., Wiley, Chichester, England, 2001, pp.81165

15. Kulfan B. M., and Bussoletti J. E., ““'Fundamental” Parametric Geometry Representations for Aircraft Component Shapes", 11th AIAA/ISSMO Multidisciplinary Analysis and Optimization Conference: The Modeling and Simulation Frontier for Multidisciplinary Design Optimization, AIAA—2006-6948 Washington, DC, 2006

16. Zhu F., and Qin N., "Intuitive Class/Shape Function Parameterization for Airfoils", AIAA Journal, Vol. 52, No.1, 2014, pp.17-25

17. MacMillan W.L., "Development of a Module Type Computer Program for the Calculation of Gas Turbine Off Design Performance”, PhD thesis, Department of Power and Propulsion, Cranfield University, Cranfield, England, 1974

18. Pachidis V., Pilidis P., Marinai L., and Templalexis I., "Towards a full two dimensional gas turbine performance simulator", Aeronautical Journal, Vol. 111, No. 1121, 2007, pp. 433-442

19. Salam A., and Eunus Z.I., "Recent Green Aviation Technologies an Overview", Journal of Modern Science and Technology, Vol. 1, No. 1, 2013, pp. 61-75

20. ANSYS Academic Research, Software Package, Release 15.0., Canonsburg, PA, 2014.

21. Roache P., "Perspective: A Method for Uniform Reporting of Grid Refinement Studies", Journal of Fluids Engineering, Vol. 116, No. 3, 1994, pp. 405-413

22. Christie R., MacManus D., and Ramírez S., "Aero-engine installation modelling and the impact on overall flight performance", Advanced Aero Concepts, Design and Operations: Applied Aerodynamics Conference, London, 2014

23. Ministry-Industry Drag Analysis Panel (MIDAP) Study Group, "Guide to in-flight thrust measurement of turbojets and fan engines", AGARD, Report AGARD-AG-237, AGARDograph No. 237, 1979

24. Shevell R.S., and Bayan F.P., "Development of a method for predicting the drag divergence Mach number and the drag due to compressibility for conventional and supercritical wings", Department of Aeronautics and Astronautics, Stanford University, Technical Report, SUDAAR-522, Stanford, CA, 1981

25. Zitzler E., and Thiele L., "Multiobjective Optimization Using Evolutionary Algorithms - A Comparative Case Study", Parallel Problem Solving from Nature Proceedings of the $5^{\text {th }}$ Workshop, PPSN V, Berlin, 1998, pp. 292-301

26. While L., Bradstreet L., Barone L., WFG 1.11, Walking Fish Group, Software, Crawley, Australia, 2015 
2017-01

\section{Short and slim nacelle design for ultra-high BPR engines}

Robinson, M.

American Institute of Aeronautics and Astronautics

Matthew H. Robinson, David G. MacManus, Kelvin Richards, and Christopher Sheaf. "Short and slim nacelle design for ultra-high BPR engines", 55th AIAA Aerospace Sciences Meeting, AIAA SciTech Forum, (AIAA 2017-0707)

http://dx.doi.org/10.2514/6.2017-0707

Downloaded from Cranfield Library Services E-Repository 